\title{
Optimal filtering of multidimensional random fields generated by autoregressions with multiple roots of characteristic equations
}

\author{
N A Andriyanov ${ }^{1,2}, K_{\text {K Vasiliev }}^{1}$ \\ ${ }^{1}$ Ulyanovsk State Technical University, Severny Venets, 32, Ulyanovsk, Russia, 432027 \\ ${ }^{2}$ Ulyanovsk Civil Aviation Institute, Mozhaiskogo, 8/8, Ulyanovsk, Russia, 432071 \\ e-mail: nikita-and-nov@mail.ru
}

\begin{abstract}
The use of mathematical models allows to compare the theoretical expressions and simulation results. Autoregressive random fields can be used for description of the images, however, such models have pronounced anisotropy, and the simulated images are too sharp. The elimination of this drawback is possible through the use of models with multiple roots of characteristic equations. The analysis shows that using models with multiple roots in filtering images with smoothly varying brightness provides smaller errors than the use of autoregressive random fields. However, studies of the dependences of filtering efficiency on various model parameters and signal-to-noise ratios for multidimensional autoregressive random fields were almost not carried out. The article discusses the solution of the problem of optimal filtering of images based on models with multiple roots of characteristic equations. Theoretical dependences of the relative variance of the filtering error on the dimension of random fields are obtained. Furthermore, it was presented some results of filtering real images by such model in comparison with autoregressive model.
\end{abstract}

\section{Introduction}

Currently there are many different mathematical models of random fields (RF) using for describing images [1-5]. The popularity of this approach is due to a number of advantages that mathematical models provide. First of all, it is the generation of sufficiently large volumes of material for research, and also mathematical models act as a tool for developing and testing various algorithms. The simplest autoregressive models generate RF with pronounced anisotropy and such models are suitable for describing only a narrow class of real multidimensional images. Doubly stochastic models [6,7] provide a change in the probabilistic properties of the generated RF at each point, but on average the properties of such a model depend on the model chosen for the main RF simulation. Therefore, to obtain RFs that are close to isotropic fragments of multispectral images, it is necessary to use autoregression with multiple roots of characteristic equations [8-10].

However, one of the main tasks of signal processing is the noise reducing or filtering. It is often considered that the observed signal is an additive mixture of the information (useful) signal and white noise. In this paper we analyze the efficiency of spatial Wiener filtering of multidimensional autoregressive RFs with multiple roots of characteristic equations against additive white Gaussian noise background. At the same time the investigation is aimed at such models of different multiplicities, which provide equivalent correlation properties. The developed filtering algorithms can become very useful tool in solving various applied problems of image processing, among which an 
important place is occupied by the detection and localization of various objects in the image [11,12]. Furthermore filtering and segmentation tasks are of interesting [13,14].

\section{Model of a multidimensional random field and its linear filter}

The following equations are commonly used to describe a multidimensional autoregressive Gaussian RF

$$
x_{\bar{i}}=\sum_{\bar{j} \in D} \alpha_{\bar{j}} x_{\bar{i}-\bar{j}}+\sigma_{x} \beta_{0} \xi_{\bar{i}}, \bar{i} \in \Omega \text {, }
$$

where $X=\left\{x_{\bar{i}}, \bar{i} \in \Omega\right\} \quad$ is simulated $\mathrm{RF}$ defined on $N$-dimensional grid $\Omega=\left\{\bar{i}=\left(i_{1}, i_{2}, \ldots i_{N}\right): i_{k}=1 \ldots M_{k}, k=1 \ldots N\right\} ; \quad\left\{\beta_{0}, \alpha_{\bar{j}}, \bar{j} \in D\right\}$ are coefficients of the model; $\left\{\xi_{\bar{i}}, \bar{i} \in \Omega\right\}$ is RF of random values with Gaussian distribution having zero mathematical expectation, and its variance is equal to one; $\sigma_{x}^{2}$ is variance of $\mathrm{RF}$ $x_{\bar{i}} ; D \subset \Omega$ is causal region of local states.

For such a model it is easy to find the transfer function of a linear filter. Using Z-transformation for model (1) it is possible to get a spatial linear filter, which is described by the transfer function of the following form

$$
H(\bar{z})=\frac{\sigma_{x} \beta_{0}}{1-\sum_{\bar{j} \in D} \alpha_{\bar{j}} \bar{z}^{-\bar{j}}},
$$

where $\bar{Z}^{-\bar{j}}=z_{1}^{-j_{1}} Z_{2}^{-j_{2}} \ldots z_{N}^{-j_{N}}$.

It should be noted that the transfer function (2) also depends on the parameters of the signal model, as does the energy spectrum of such a RF. The relationship of the transfer function (2) and the energy spectrum of the RF $X$ is determined by the expression

$$
S_{x}(\bar{Z})=H(\bar{Z}) H\left(\bar{Z}^{-1}\right) .
$$

The analysis of probabilistic properties of the RF is simplified if the transfer function of a multidimensional filter can be factorized: $H(\bar{z})=\prod_{k=1}^{N} H_{k}\left(z_{k}\right)$. Then the energy spectrum $S_{x}(\bar{z})=\prod_{k=1}^{N} S_{k}\left(z_{k}\right)$ and correlation function (CF) $B(\bar{r})=\prod_{k=1}^{N} B_{k}\left(r_{k}\right)$ are also can be factorized. Simple and very useful for applications multidimensional splittable RF $x_{\bar{i}}$ can be represented using spatial autoregression

$$
\prod_{k=1}^{N}\left(1-\rho_{k} z_{k}^{-1}\right)^{m_{k}} x_{\bar{i}}=\sigma_{x} \beta_{0} \xi_{\bar{i}}, \bar{i} \in \Omega,
$$

with multiple roots $\rho_{k}$ of characteristic equations having multiplicities $m_{k}, k=1,2, \ldots, N$.

The transfer function of such a RF will be factorizable and will be written as

$$
H(\bar{z})=\sigma_{x} \beta_{0} / \prod_{k=1}^{N}\left(1-\rho_{k} z_{k}^{-1}\right)^{m_{k}},
$$

where $\beta_{0}=\prod_{k=1}^{N} \beta_{k} ; \beta_{k}\left(m_{k}\right)=\left(1-\rho_{k}^{2}\right)^{2 m_{k}-1} / \sum_{l=0}^{m_{k}-1}\left(C_{m_{k}-1}^{l} \rho_{k}^{l}\right)^{2}, C_{j}^{i}=j ! /(i !(j-i) !)$.

\section{Filtering efficiency of multidimensional random fields with multiple roots of characteristic equations}

One of the difficult tasks of filtering image sequences on multidimensional grids is the analysis of the effectiveness of such filtering. In this case, the necessary criterion for analysis is the dependence of the variance of the filtering error on various model parameters and noise. Formally, spatial covariance 
matrices of estimation errors can be calculated using the recurrence relations for the Kalman filter [6,7]. However, if it is necessary to compare the algorithms for different values of the parameters of the stochastic equations and noise levels, the determination of even steady-state values of the elements of the covariance matrices becomes a very laborious task.

Consider a relatively simple way to determine the effectiveness of estimating homogeneous fields on infinite grids based on the basic principles of Wiener's filter theory [8]. Using the observations $z_{\bar{j}}=x_{\bar{j}}+n_{\bar{j}}, \bar{j}=\left(j_{1} j_{2} \ldots j_{N}\right)^{T} \in \Omega$, which are the sum of informational (useful) RF and additive white Gausian noise with a variance $\sigma^{2}=M\left\{n_{\bar{j}}^{2}\right\}$ it is necessary to make the best (in the sense of the minimum error variance) linear estimate $\hat{x}_{\overline{0}}=\sum_{\bar{j} \in \Omega} h_{\bar{j}} z_{\bar{j}}$ of element $x_{\overline{0}}$ in informational RF. This estimation will use coefficients $h_{\bar{j}}$ which will determine the optimal filtering. The search of minimum error variance $\sigma_{\varepsilon}^{2}=M\left\{\left(\hat{x}_{\overline{0}}-x_{\overline{0}}\right)^{2}\right\}=M\left\{\left(\sum_{\bar{j} \in \Omega} h_{\bar{j}} z_{\bar{j}}-x_{\overline{0}}\right)^{2}\right\}$ can be written as a system of linear equations

$$
h_{q} \sigma^{2}+\sum_{\bar{j} \in \Omega} h_{\bar{j}} B(\bar{r}-\bar{j})=B(\bar{r}), \quad \bar{r} \in \Omega,
$$

which can be considered as a spatial analogue of the Wiener-Hopf equations.

Using multidimensional $\mathrm{Z}$-transformation it is possible to find equations system solution and expression for the relative error variance [8]:

$$
\frac{\sigma_{\varepsilon}^{2}}{\sigma_{u}^{2}}=\frac{1}{(2 \pi)^{N}} \int_{-\pi}^{\pi} \ldots \int_{-\pi}^{\pi} \frac{\beta_{0}^{2}}{\prod_{k=1}^{N}\left(1+\rho_{k}^{2}-2 \rho_{k} \cos \lambda_{k}\right)^{m_{k}}+q \beta_{0}^{2}} d \bar{\lambda} .
$$

where $q=\sigma_{x}^{2} / \sigma^{2}$ is signal-to-noise ratio, $N$ is the dimension of RF, $m_{k}$ is the model's multiplicity for $k$-th dimension, $\rho_{k}$ is correlation parameter in $k$-th dimension.

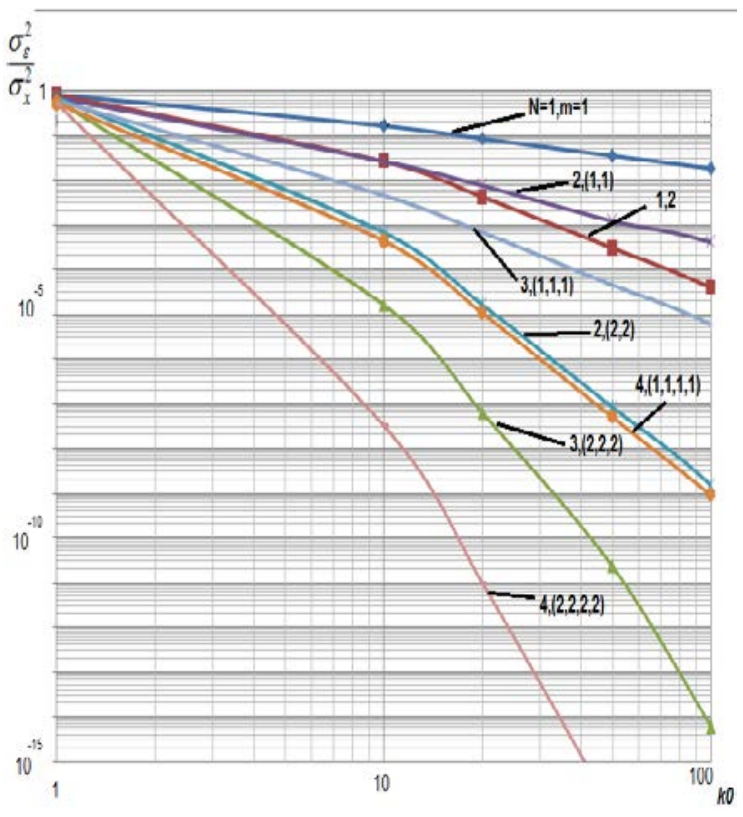

a)

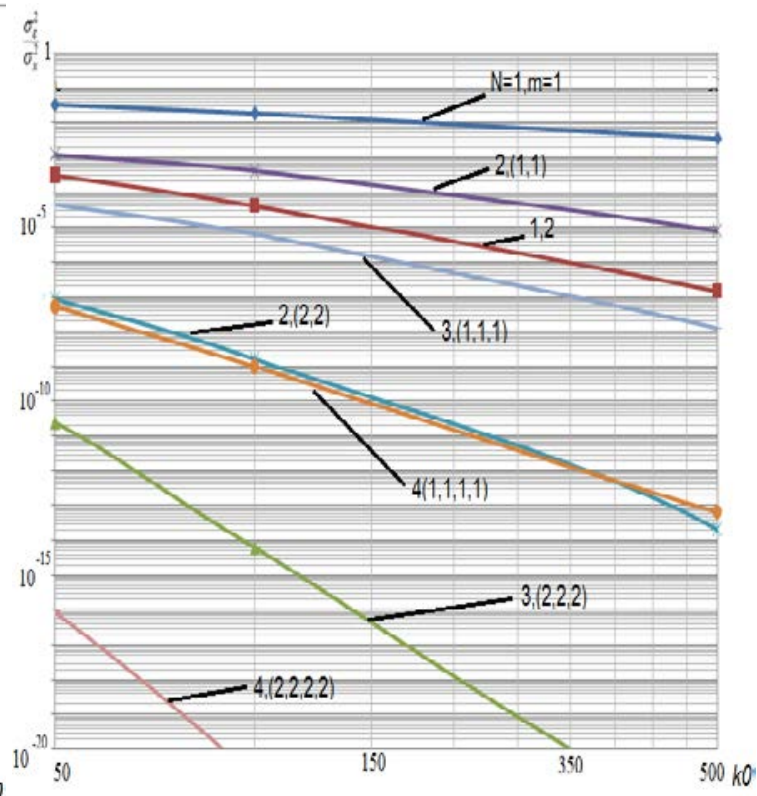

b)

Figure 1. Relative variances of errors of multidimensional RF. 
Figure 1 shows the dependence of the relative error of the filter variance on the correlation interval $k_{0}$ for models of different dimensions and orders with $q=0.1$. Correlation interval is less than 100 in figure 1a and less than 500 in figure 1b. This interval determines the equivalence of models of different multiplicities. On the graphs, the dimension is indicated as $N$, the multiplicity as $m$.

The analysis of the obtained dependences shows that with sufficiently small correlation intervals $\left(k_{0}<10\right)$ the variances of filtering errors of autoregressive RF of the 1st and 2nd orders are rather close. An increase in the dimensions and a further increase in the correlation interval leads to the distancing of the curves. At the same time the smallest values of relative variances of filtering errors are obtained for the cases $N=3, m=(2,2,2), N=4, m=(2,2,2,2)$. This is because when $m=1$ autoregression along the axes are quite prickly and their filtering is a more difficult task.

Using expression (7), one can obtain the following equations for models of various dimensions, presented in Table 1.

Table 1. Filtering efficiency of RFs of different dimensions.

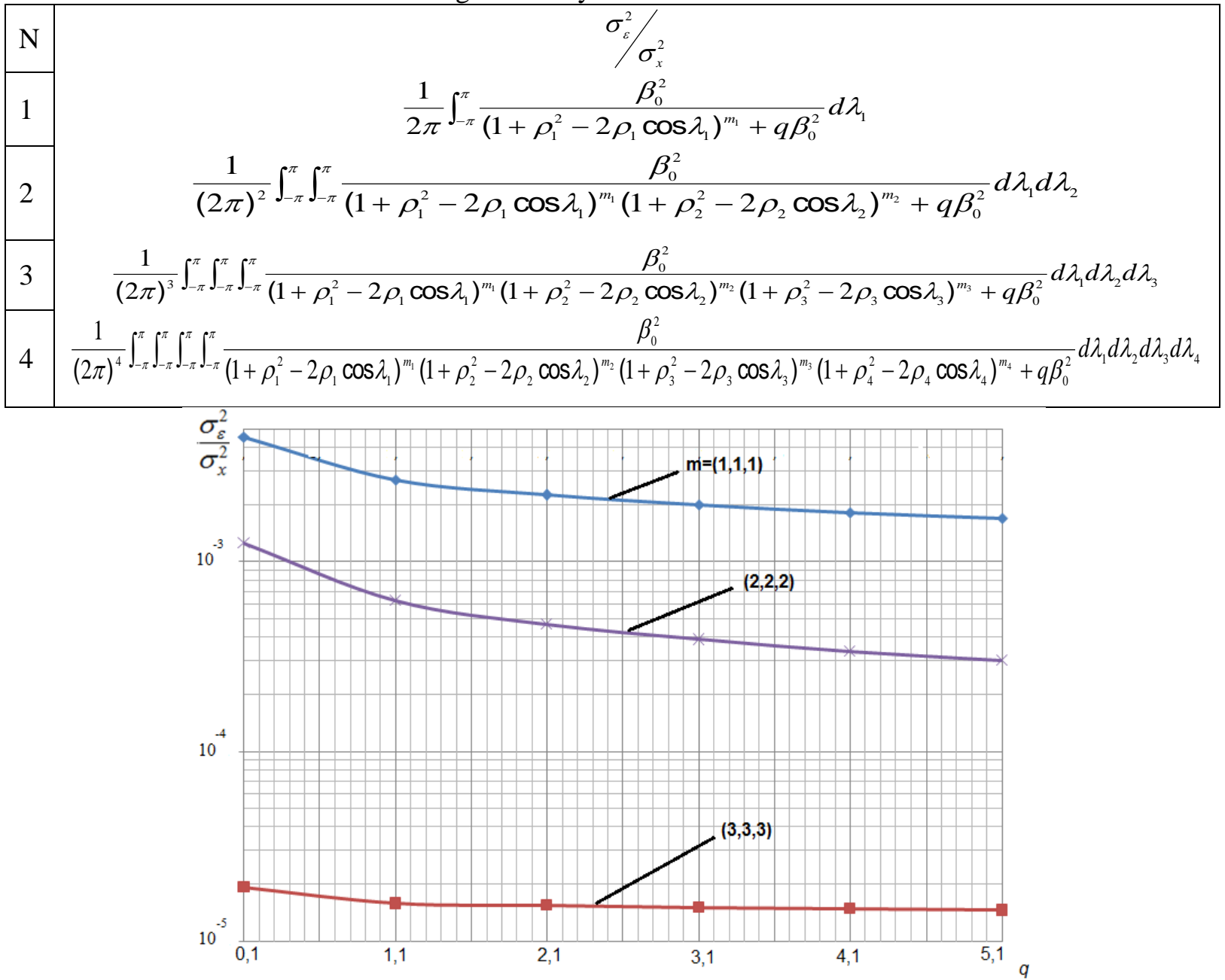

Figure 2. Relative variances of filtering errors for three-dimensional RF.

Figure 2 shows the dependences of the relative error of filtering variance on the signal-to-noise ratio q for models of three-dimensional $\mathrm{RF}$ of different orders with $k_{0}=50$.

An analysis of the curves in the graph shows that in the case of a large dimension of the RF, for example $\mathrm{N}=3$, the variance of the filtration error is rather small. In this connection, effective filtering is obtained both for small signal-to-noise ratios and for large ones. At the same time, an increase in the multiplicity of models leads to a decrease in the relative dispersion of the filtering error. 
Figure 3 shows the dependences of the relative variance of filtering errors on the dimension of the AR for the cases $k 0=100, q=0.01$ with multiplicities $m=1$ and $m=2$.

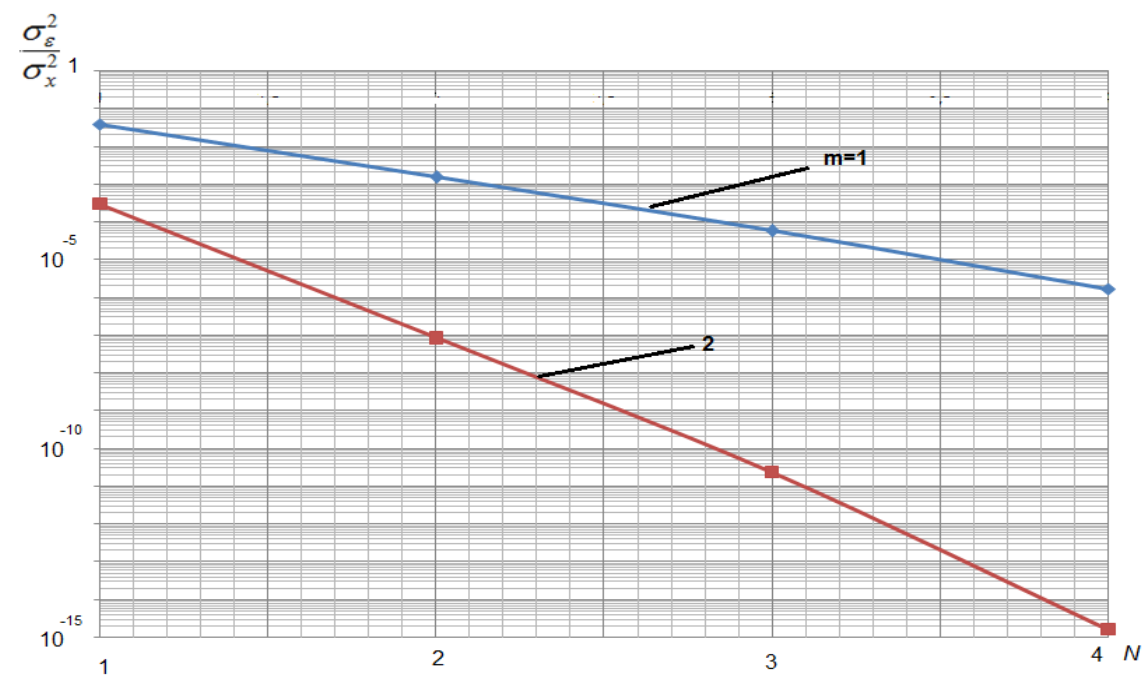

Figure 3. Dependence of the relative error variance on the dimension.

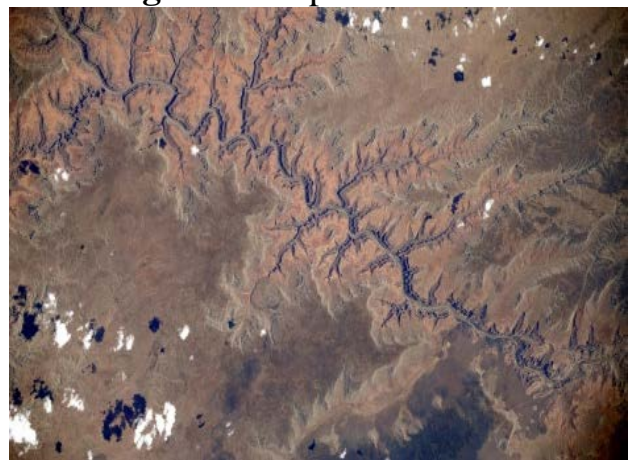

a)

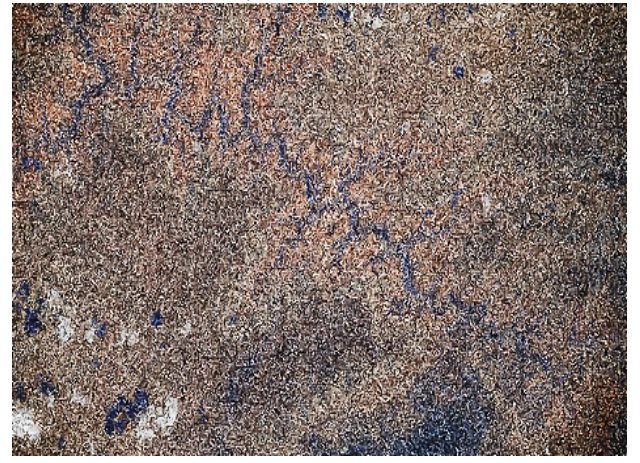

c)

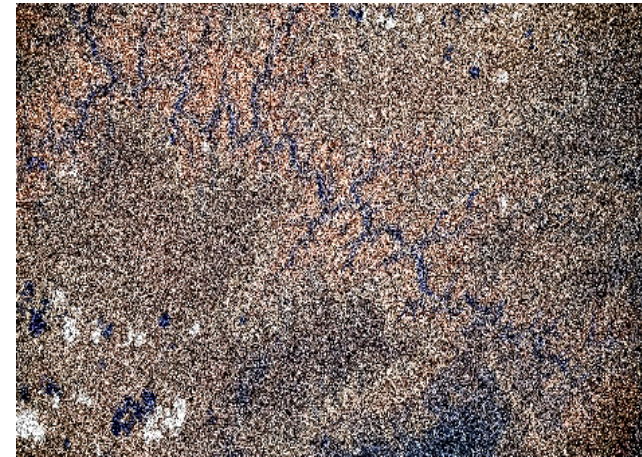

b)

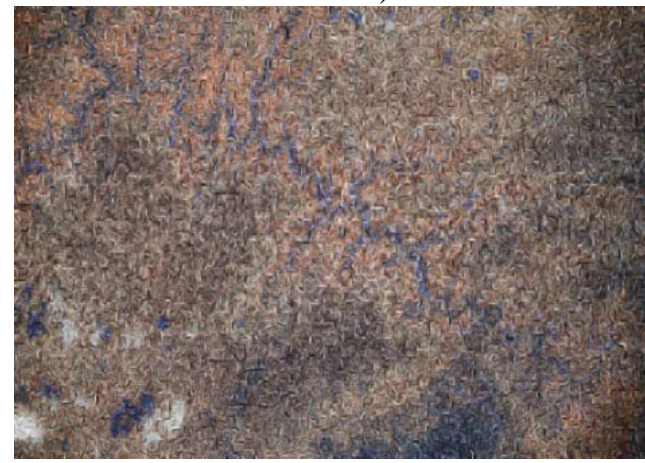

d)

Figure 4. Filtering of satellite images.

The analysis of the curves presented in Figure 3 shows that increasing the dimension of the RF leads to a significant increase in filtration efficiency, which is associated with a large number of correlations in the multidimensional model. At the same time large dimensions provide variance of filtering errors tending to $0\left(\sim 10^{-15}\right)$ already with multiplicities $m=2$ along each axis. At the same time if $m=1$ then the variance of the filtering error is several orders of magnitude greater.

\section{Real image processing}

The filtering algorithm based on a multiple-root model was tested on a multidimensional satellite image compared to an algorithm based on autoregressive models. Figure 4 shows the filtering results 
for one of the images. Figure 4a shows the source image, figure $4 \mathrm{~b}$ shows the noisy image, figure 4c shows the filtering results using autoregressive model of the first order and figure $4 \mathrm{~d}$ shows the filtering results using autoregressions with multiple roots model.

The analysis of the presented pictures shows the model with multiple roots provides better results in variance of filtering error, for example, the results for image on figure 4 is following: relative error variance for figure $4 \mathrm{c}$ is 0.782 , error variance for figure $4 \mathrm{~d}$ is 0.358 . The signal-to-noise ratio is 0.5 .

\section{Conclusion}

Thus, in this paper, the filtration efficiency of multidimensional RF with multiple roots of characteristic equations is investigated. At the same time, an increase in the dimensions and orders of the models leads to a significant decrease in the relative dispersion of filtering error. Therefore, it is advisable to use less computationally sophisticated mathematical models of RFs that provide fairly small errors. For example, already for the dimension $N=3$ it is possible to achieve relative error equal $10^{-5}$ for $q=0.01$ and multiplicities $m=(3,3,3)$. In addition, studies have been conducted on the effectiveness of filtration depending on the dimension of the RF. It should be noted that in the logarithmic axes, these dependencies are close to linear for the dimensions $N=1, \ldots, 4$. Such models are also useful in processing real images having strong correlation properties.

\section{References}

[1] Ohtsu K, Peng H and Kitagawa G 2015 Time Series Analysis Through AR Modeling Time Series Modeling for Analysis and Control (Springer Briefs in Statistics. Springer, Tokyo) 7-56

[2] Aue A, Horvath L 2011 Quasi-Likelihood Estimation In Stationary And Nonstationary Autoregressive Models With Random Coefficients Statistica Sinica 21 973-999

[3] Praline E Yu 2017 Trends in the processing of random fields in modeling Collection of scientific works based on proceedings of conference New paradigm of science and education: on the way to the convergence of knowledge, technology 72-73

[4] Vasil'ev K K, Dement'ev V E and Andriyanov N A 2015 Doubly stochastic models of images Pattern Recognition and Image Analysis. Advances in Mathematical Theory and Applications 25(1) 105-110

[5] Bolin, D 2012 Models and Methods for Random Fields in Spatial Statistics with Computational Efficiency from Markov Properties (Centre for Mathematical Sciences, Mathematical Statistics, Lund University) 118-126

[6] Vasiliev K, Dementiev V and Andriyanov N 2018 Representation and processing of multispectral satellite images and sequences Procedia Computer Science 126 49-58 DOI: 10.1016/j.procs.2018.07.208

[7] Andriyanov N A 2018 Software Complex for Representation and Processing of Images with Complex Structure CEUR Workshop Proceedings 2274 10-22

[8] Andriyanov N A, Vasiliev K K 2018 Use autoregressions with multiple roots of the characteristic equations to image representation and filtering CEUR Workshop Proceedings 2210 273-281

[9] Vasiliev K K, Andriyanov N A 2017 Analysis of autoregression with multiple roots of characteristic equations Radiotekhnika 6 13-17

[10] Vasiliev K K, Andriyanov N A and Abdulkadim Kh A 2018 Filtration efficiency of random fields with multiple roots of characteristic equations Radiotekhnika 6 20-23

[11] Ilyasova N A, Paringer R, Shirokanev A, Kupriyanov A and Ushakova N 2017 Smart feature selection technique for object localization in ocular fundus images with the aid of color subspaces Procedia Engineering 201 736-745

[12] Andriyanov N A, Vasiliev K K and Dementiev V E 2017 Anomalies detection on spatially inhomogeneous polyzonal images CEUR Workshop Proceedings 1901 10-15 DOI: 10.18287/1613-0073-2017-1901-10-15

[13] Filimonov P A, Belov M L, Fedotov Yu V, Ivanov S E and Gorodnichev V A 2018 Aerosol 
inhomogeneity segmentation algorithm Computer Optics 42(6) 1062-1067 DOI: 10.18287/2412-6179-2018-42-6-1062-1067

[14] Chervyakov N I, Lyakhov P A and Orazaev A R 2018 Two methods of adaptive median impulse noise filtering on images Computer Optics 42(4) 667-678 DOI: 10.18287/2412-61792018-42-4-667-678

\section{Acknowledgement}

The study was supported by RFBR, Project №17-01-00179. 